\title{
STEPS TOWARD THE MOON-BASED ASTRONOMY PLANNING IN JAPAN
}

\author{
N.KAIFU \\ National Astronomical Observatory of Japan
}

\begin{abstract}
Lunar surface is, in spite of disadvantages on payload compared with those in the distant orbits, extremely attractive for future astronomical observations with large and complicated observing instruments. The Moon will provide firm and large base, very stable temperature and low background in some selected sites. In addition, the aids of human activities on the Moon can be expected in the near future. We have been discussing the small but realistic steps toward the future Moon-based astronomy, as part of Japanese lunar exploration plans with HI rockets. The fundamental Policy of Japan's Space Development which was approved by Space Activities Commission in 1996 described that Japan will continuously promote unmanned lunar exploration and study the feasibility of a systematic exploration program for science and possible activities on the Moon, under a phase-by-phase approach. The plan includes a possibility of astronomical observations on the lunar surface.

Some pilot-type astronomical plans, starting from very small-size telescopes in optical/IR and $\mathrm{mm}$-wave etc. are under discussion. We report here on the general background, discussion including proposed plans, and steps toward the future moon-based observatories.
\end{abstract}

\section{Background of the Study}

1994 The Special Committee on Long-Term Vision reported "Japan's Space Long-Term Vision".

1996 "Fundamental Policy of Japan's Space Development" by Space Activities Commission containing the following items was released;

$\diamond$ continuously promote unmanned lunar exploration,

$\diamond$ study systematic exploration program for science and possible activities on the Moon,

$\diamond$ future astronomical observatory on the lunar surface,

$\diamond$ cooperation of NASDA, ISAS, NAOJ and other space science research institutes,

$\diamond$ unmanned astronomical observatory, 2010 and after,

$\diamond$ possible manned activities on the Moon through international cooperation, 2020 and after.

Relating above movements, the following studies of Moon-based astronomy has been made:

1992 93: Study of movable optical interferometer, by Moon and Planets Association (chair: Karoji, H.).

1994 95: Preliminary study of science on the Moon, by Institute for Future Technology (chair: Mizutani, H., astronomy section: Kaifu, N.) : informal study of astronomy on the Moon, by LUNARIAN (organized by Kaifu, N.). 
1994 : The first version of RISE (Radio Interferometry for Selenodesy, project leader: Kawano, N.) project started as a part of SELENE (NASDA-ISAS Lunar-orbiter) for 2003.

1995 : Further study of astronomy on the Moon by Institute for Future Technology, and by LUNARIAN (chair: Kaifu, N.) : proposed a pilot telescope plan on the Lunar high-latitude site for the 2nd HII mission, and other instruments for further missions.

\section{General Considerations}

The Moon as the site of astronomical observations can be summarized as follows.

[noise] Good, but depends on the site. No atmospheric noise. Scatter and emission from the Sun, the Earth and the lunar soil can be avoided in selected areas such as permanent shadows and anti-earth surface.

[heat] Good or poor, depending on the site. Solar radiation and heat conduction from the lunar soil can be almost completely avoided in the permanent shadow areas.

[foundation] Excellent. Farm and large flat areas with low vibration are available.

[payload] Poor. Nearly $1 / 2$ available payload compared with distant orbits. This is the most serious disadvantage compared with orbiting satellite observatories.

infrastructure] Poor/good/excellent, depending on location and time scale. The energy supply is difficult in night time, but some locations could be free from this difficulty. The problem will be much easier in the future when the human activities on the Moon are expected.

Considering the above-mentioned summary, we find that the Moon can provide us with excellent sites for future astronomical observations. Preferable sites for the Moon-based astronomy are as follows:

\section{[Polar Crater (permanent shadow) Site]}

There exist such a crater according to the CLEMENTINE data. The permanent shadow craters provide;

$\diamond$ very stable thermal condition with almost no temperature change,

$\diamond$ very low background noise by low ambient temperatures (70K or lower) and by shielding of radiation from the Sun and the Earth,

$\diamond$ excellent energy supply via nearby high peak with permanent Sun-shine,

$\diamond$ good communication link via nearby high peak with permanent Earth-view.

The permanent shadow polar crater site is ideal for various astronomical observations, especially for interferometers from optical to radio wavelengths with long $(1-20 \mathrm{~km})$ base lines. However, the accessibility is not good for short time range, and we need a step-by-step approach toward this very attractive observation site.

\section{[High-Latitude (80 90 deg) Site]}

A good practical step toward the permanent shadow is to go to the high altitude site with Sun-shine in daytime. Where we can expect;

$\diamond$ good accessibility,

$\diamond$ easier heat/noise reduction compared with the equatorial region.

Therefore the high-latitude sites are attractive for pilot-phase astronomy in the early-phase explorations.

\section{[Anti-Earth Surface Site]}


Because of the increasing artificial radio signals as well as the strong ionospheric radiation, the anti-earth surface of the Moon is important by;

$\diamond$ low background noise, especially in low frequency radio waves.

The future low-frequency radio astronomy and SETI will find attractive sites in the equatorial area of the anti-earth side.

To summarize, the Moon is a very attractive site for future astronomical observations, especially for long base line interferometers of optical, IR, sub-mm and gravitational waves etc. in the polar craters, and for SETI and low frequency radio arrays on the anti-earth-surface.

Difficulty for near future activity on the Moon is costliness, and careful steps are required to approach future "Lunar Observatories". However, there are driving factors toward the Moon. The Moon is still a place of dream for humankind which will provide the base for the first stage of "real" human space activities in the early 21st century. By the aid of human activities which will follows the "non-man" explorations, the astronomy from the Moon would quickly be developed.

\section{Proposed Plans and Discussion of Lunar-Based Astronomy}

The following plans of astronomical observations from the Moon have been proposed and discussed in the course of various studies of Astronomy on the Moon by using HII Rockets, as described in section 1 .

\subsection{EARLY-PHASE EXPLORATION (2003 2015)}

\section{(First Exploration Launch)}

Accepted as a Lunar Orbiter "SELENE", for 2003 launching. Cooperating project by NASDA and ISAS. It includes a VLBI observation project "RISE" supported by NAOJ.

RISE: Radio Interferometry for Selenodesy, PI; Kawano, N. (NAOJ).

Stable radio emitters on the lunar orbiter and lander to measure the accurate orbit movement of lunar surface by means of relative VLBI.

Related Instruments: Laser Altimeter, Radar Altimeter Sounder, Relay satellite, etc.

$\diamond$ Explore the sites for astronomical observations.

\section{(Second Exploration Launch)}

Under consideration. Could be a combination of penetrator network and a test lander with a small IR/Optical Telescope "PILOT".

$\diamond$ PILOT: Pilot Lunar Optical-IR Telescope, a test plan by NAOJ, NASDA and MELCO A $30 \mathrm{~cm}$ class simple and light-weight telescope, with high-sensitivity IR and visual images, to test the observational abilities of astronomy and NEO from the highlatitude site of the Moon.

aim: test astronomical observations from high-latitude site of the Moon, and obtain some good scientific data in the near IR and in the visual region.

aperture: $30 \mathrm{~cm}$ (light-weighted mirror).

detectors: visual $4 \mathrm{~K} \times 4 \mathrm{~K}$, FOV 22', 0.3 " / pix \& NIR $1 \mathrm{~K} \times 1 \mathrm{~K} \times 3$ (multi-color) and may have spectroscopic capability.

site: high-latitude ( $85-90$ deg south). Average surface temperature at noon is estimated to be $215 \sim 220 \mathrm{~K}$ at 85 deg site.

weight : $100 \mathrm{~kg}$ including control system, sun shield, etc.

\section{(Proposals/Plans for third and later Exploration Launches)}

For the third and later Exploration Launches we consider simple and single telescope systems which can be carried by single HII lander and we can provide valuable new scientific achievements. 
$\diamond$ MM-Wave Cosmic Background Survey, proposed by Tsuboi, M.

An 1.5-m size ultra-low noise antenna for $30-90 \mathrm{GHz}$ at high-latitude site, to measure the 10' scale faint structure of cosmic background radiation. Details will be discussed seperately in this JD by Tsuboi.

$\diamond$ IR Compact Telescope, proposed by Ueno, M. and Shibai, H.

An 80-cm aperture, 100-K telescope with IR imager and prism spectrometer of 1-5 $\mathrm{mm}$ on the high-latitude site, mainly for high-accuracy measurements of galaxies.

$\diamond$ NEO Monitoring Telescope, proposed by Isobe, S.

Twin 35-cm telescopes (first phase) and twin 1-m telescopes (second phase).

\subsection{SECOND-PHASE EXPLORATION (2015-2030?)}

In this phase we assume the activities by unmanned simple observatories, with (possible) some human aids.

$\diamond$ IR Interferometer for Astronomy, proposed by Nishikawa, J.

A $25-\mathrm{cm} \times 2,1-\mathrm{km}$ base line interferometer at $2-\mathrm{mm}$ in polar site, to measure 1 marcsec $=(5 \mathrm{~km} / \mathrm{s})$ year $/ \mathrm{Mpc}$.

$\diamond$ Gravitational Wave Telescope, proposed by Ohashi, M.

A $50-\mathrm{km}$ base line, simple Michelson interferometer $50-\mathrm{cm}$ diameter mirrors in the polar crater, for the 1-100 $\mathrm{Hz}$ wave (merging black holes and neutronstar pulses).

$\diamond$ Low Frequency Radio Array and SETI, proposed by Hirabayshi, H. and Ishiguro,M.

\section{Toward a Future Lunar-Based Observatory}

To realize the outstanding astronomical observations from the Moon in the far future, we need to promote realistic and continuous activities. The following items are important:

[1] Public Understanding: The value of astronomy from the Moon should be clearly shown to the public.

[2] Step-by-Step planning: Continuous promotion of realistic planning, development of technology, development of good sites, and good results including science.

[3] Future Human Activities Little human activity can easily exceed the sophisticated automatic system, and will reduce the cost in the future

[4] International Cooperation: Definitely important, though depends on the phase of exploration. 